\title{
Interferometric Space Missions for Exoplanet Science: Legacy of Darwin/TPF
}

\author{
D. Defrère ${ }^{1}, \mathrm{O}$. Absil $^{1}$, and C. Beichman ${ }^{2}$
}

${ }^{1}$ Space sciences, Technologies \& Astrophysics Research (STAR) Institute, University of Liege, Liege, Belgium.

${ }^{2}$ NASA Exoplanet Science Institute, California Institute of Technology, Jet Propulsion Laboratory, Pasadena, California, USA.

\begin{abstract}
DARWIN/TPF is a project of an infrared space-based interferometer designed to directly detect and characterize terrestrial exoplanets around nearby stars. Unlike spectro-photometric instruments observing planetary transits, an interferometer does not rely on any particular geometric constraints and could characterize exoplanets with any orbital configuration around nearby stars. The idea to use an infrared nulling interferometer to characterize exoplanets dates back to Bracewell (1978), and was extensively studied in the 1990s and 2000s by both ESA and NASA. The project focuses on the mid-infrared regime $(5-20 \mu \mathrm{m})$, which provides access to key features of exoplanets, such as their size, their temperature, the presence of an atmosphere, their climate structure, as well as the presence of important atmospheric molecules such as $\mathrm{H}_{2} \mathrm{O}, \mathrm{CO}_{2}, \mathrm{O}_{3}, \mathrm{NH}_{3}$, and $\mathrm{CH}_{4}$. This wavelength regime also provides a favorable planet/star contrast to detect the thermal emission of temperate $(\sim 300 \mathrm{~K})$ exoplanets $\left(10^{7}\right.$ vs $10^{10}$ in the visible). In this chapter, we first review the scientific rationale of a mid-infrared nulling interferometer and present how it would provide an essential context for interpreting detections of possible biosignatures. Then, we present the main technological challenges identified during the ESA and NASA studies, and how they have progressed over the last 10 years. Finally, we discuss which technologies remain to be developed before flying such an instrument, and possible ways to make DARWIN/TPF a reality in the mid-term future.
\end{abstract}

\subsection{Introduction}

The quest for other habitable worlds and the search for life among them are major goals of modern astronomy. Shortly after the announcement of the first unambiguous detection of a planet around a star other than the Sun (Mayor and Queloz 1995), mission concepts capable of meeting these challenges were proposed to both ESA and NASA. In Europe, ESA focused mainly on the DARWIN project (Léger et al 1996a,b; Fridlund 2000, 2004; Fridlund et al 2006), which consisted of a space-based flotilla of mid-infrared $(6-20 \mu \mathrm{m})$ telescopes using nulling interferometry as measurement principle. In the United states, a similar concept, called the terrestrial planet finder interferometer (TPF-I, Angel et al 1997; Woolf and Angel 1998; Beichman et al 1999; Beichman 2000), was considered as the final piece of NASA's ambitious Navigator program to characterize Earth-like exoplanets (Lawson and Traub 2006). Between 1996 and 2007, considerable efforts have been carried out by both agencies to define the best mission design and to advance the technologies required for such an ambitious endeavor. Several mission architectures were proposed, key enabling technologies were developed and demonstrated on laboratory test-benches, and advanced data reduction techniques were investigated. These efforts, which resulted in hundreds of articles in the technical literature, culminated in 2007 with the convergence and consensus on a mission architecture called the Emma X-array. In parallel, both agencies also appointed teams to investigate the scientific issues related to the search for life on exoplanets (e.g., Lawson et al 2007; Fridlund et al 2010). What is a biosignature? What is the minimum number of stars to observe during the mission lifetime? What is a good target catalog? were a few important questions addressed by these teams.

While most DARWIN and TPF-I activities stopped after 2007, the main scientific questions that the missions were supposed to address remain unanswered to this day: What is the atmospheric composition of rocky exoplanets? Are they habitable? Do they show signs of biological activity? How common is a planet like Earth? How do they form? Today, the exoplanet landscape has greatly changed compared to 2007 but the general consensus in the exoplanet community is still the same: mid-infrared spectra will be required to tackle these fundamental questions. In addition to providing a favorable planet/star contrast to detect the thermal emission of exoplanets (see Figure 0.1), the midinfrared regime provides data to measure key details about planets, such as their size, their temperature, the presence 


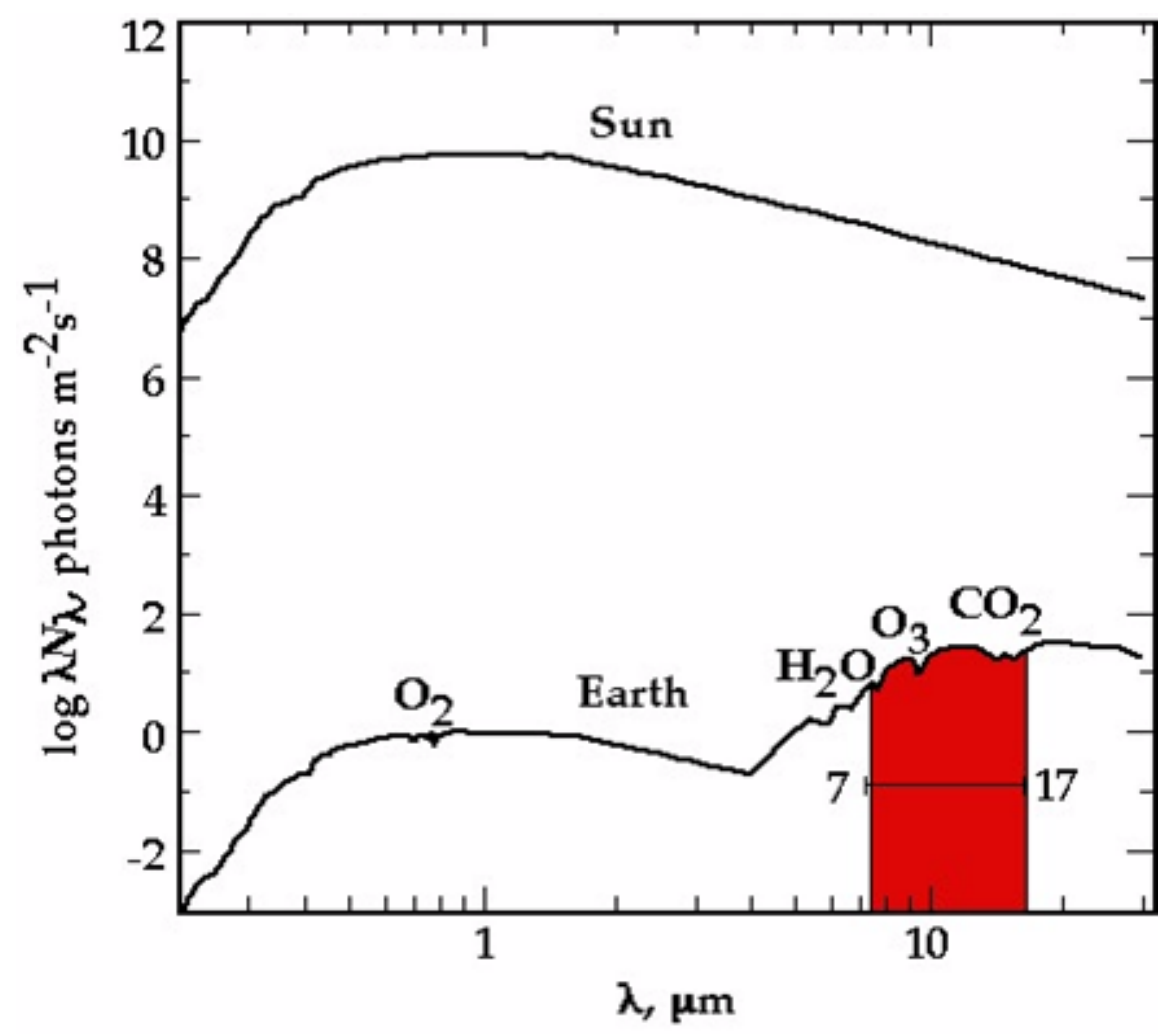

Fig. 0.1: The photon flux from the Sun and the Earth as seen from a distance of $10 \mathrm{pc}$ from UV/visible to mid-IR wavelengths (figure from Beichman et al 1999). The Sun-Earth contrast ratio is a factor of $\sim 1000$ more favorable at infrared wavelengths than in the visible which makes the starlight suppression easier at longer wavelengths. The presence of broad spectral markers in the Earth's atmosphere, including $\mathrm{H}_{2} \mathrm{O}, \mathrm{O}_{3}$, and $\mathrm{CO}_{2}$ also make the mid-infrared a valuable wavelength regime.

of an atmosphere, their climate structure, as well as the presence of important atmospheric molecules such as $\mathrm{H}_{2} \mathrm{O}$, $\mathrm{CO}_{2}, \mathrm{O}_{3}$, and $\mathrm{CH}_{4}$.

In this chapter, we present the DARWIN/TPF-I project, from its scientific goals to its instrumental requirements and challenges. In the first part of this chapter, dedicated to science, we discuss the relevance of mid-infrared observations for better understanding planetary atmospheres and explain why it has a key role to play in the search for life beyond Earth in today's exoplanet landscape. In the second part of this chapter, we review the main difficulties associated with the study of rocky planet atmospheres and show how nulling interferometry can tackle these challenges. The measurement principle, mission architecture, and expected performance of the DARWIN/TPF-I project are presented together with the main technological developments achieved since 2007. Finally, we illustrate the mission concept with simulated observations of the potentially habitable planet recently detected around our nearest neighboring star (Proxima Cen, Anglada-Escudé et al 2016).

\subsection{The need for multiple techniques to study exoplanets}

The goal of finding habitable planets and even planets with signs of (primitive) life is a challenging one requiring a variety of complementary observations (Beichman et al 2007). The detection of reflected light at visible wavelengths with a coronagraphic telescope (TPF-C, Traub et al 2006) would provide information on a planet's orbit as well as on the presence and composition of a planetary atmosphere. But visible light data yields no information on the planet's size or mass. However, as described below, the combination of infrared data from DARWIN/TPF-I with visible data would reveal the planet's temperature, albedo and radius as well as additional information on atmospheric composi- 
tion. But neither direct detection technique provides information on the planet's mass which must come from either micro-arcsecond astrometry (Shao et al 2007) or from precision radial velocity (Fischer et al 2016). It will be from the combination of these datasets that we will be able to remove the uncertainties and degeneracies in any one dataset to be able make a confident assessment of the planet's habitability and of the presence of life.

It is worth noting that the study of transiting systems is highly promising for the study of planets larger than a few earth radii and will be a major focus for the James Webb Space Telescope (Beichman et al 2014). But the rarity of planetary systems suitably aligned to produce a transit, the short duration of the transit, and the noise produced by the host star means that the study of the atmospheres of terrestrial planets, especially those orbiting Sun-like stars, is probably unachievable via this technique. Thus techniques which separate the light of the planet from the glare of the host star are essential. In what follows we discuss in detail the scientific return and the technical challenges of infrared observations as developed for the DARWIN/TPF-I missions.

\subsection{Scientific objectives}

\subsubsection{Searching for biosignatures}

The key scientific objectives of DARWIN/TPF-I were to detect rocky exoplanets, perform their spectral analysis, characterize their habitability, and ultimately search for signs of biological activity (i.e., biosignatures) on the most interesting candidates. A biosignature can be defined as "an observable feature of a planet, such as its atmospheric composition, that our present models cannot reproduce when including the abiotic physical and chemical processes we know about" Léger et al (2011). Searching for biosignatures by remote sensing would not be a first. Actual observations of Earth taken by the Galileo probe in 1990 showed a large amount of $\mathrm{O}_{2}$ (at $0.76 \mu \mathrm{m}$ ) with traces of $\mathrm{CH}_{4}$ (at $3.3 \mu \mathrm{m}$ ), which together were considered as strongly suggestive of biology by Sagan et al (1993). This conclusion was based on two founding papers (Lovelock 1965; Lederberg 1965), which reported that atmospheric abundances of Earth's $\mathrm{O}_{2}$ (an oxidizer associated mainly with photosynthesis) and $\mathrm{CH}_{4}$ (a reducer associated mainly with bacteria and mantle outgasing) are in large thermodynamic disequilibrium, which arises due to the presence of life. Similarly, Owen (1980) noted that the Earth's oxygen-rich atmosphere would be very difficult to be explained without biology since this highly-reactive gas would otherwise rapidly combine with the crust. Later, it was realized that a viable alternative to searching for $\mathrm{O}_{2}$ at visible wavelengths was to search for $\mathrm{O}_{3}$ in the mid-infrared $(9.6 \mu \mathrm{m}$, Angel et al 1986; Leger et al 1993; Selsis et al 2002). The latter study introduced the triple signature (i.e., $\mathrm{O}_{3}, \mathrm{CO}_{2}, \mathrm{H}_{2} \mathrm{O}$ ) as a more reliable indicator for life because it is difficult to produce $\mathrm{O}_{3}$ if water is present, due to catalytic cycles initiated by water photolysis, which remove $\mathrm{O}_{3}$. Ozone is actually a highly sensitive indicator for the existence of even a trace amount of $\mathrm{O}_{2}$ and hence easier to detect at low $\mathrm{O}_{2}$ concentrations (Des Marais et al 2002). From an observational standpoint, the possibility to look for signs of biological activity in the mid-infrared presents a huge advantage because this regime offers a more favorable planet/star contrast than the visible regime (see Figure 0.1). The DARWIN/TPF project was born from this promising possibility.

\subsubsection{A new paradigm: understanding planetary atmospheres}

The approach to search for biosignatures described in the previous section has a couple of caveats, which were already discussed but not fully realized at the time of the DARWIN/TPF-I studies: (1) false positives, where life is falsely detected, and (2) false negatives - where life is present but not found. The repercussions of these caveats are now being explored in the literature in ever greater detail and new research networks, which investigate conditions for habitability and potential atmospheric biosignatures, have recently formed across the globe to address the interdisciplinary challenge of remotely detecting life outside the Earth. In particular, other effects of biological activity on planetary atmospheres have been investigated by several authors. For instance, Segura et al (2003, 2005), Grenfell et al (2007), and Rugheimer et al (2013) considered spectral responses of gas-phase biosignatures such as $\mathrm{O}_{2}, \mathrm{O}_{3}$, nitrous oxide $\left(\mathrm{N}_{2} \mathrm{O}\right)$ and chloromethane $\left(\mathrm{CH}_{3} \mathrm{Cl}\right)$ for Earth-like planets under different conditions, e.g. orbiting different central 
stars. Kaltenegger et al (2007) investigated spectral signals for possible atmospheric compositions at different epochs in Earth history. The effect of clouds upon spectral biosignature bands was discussed by Kitzmann et al (2011). The effect of band overlap of $\mathrm{CO}_{2}$ and $\mathrm{O}_{3}$ in high $\mathrm{CO}_{2}$-atmospheres was discussed by von Paris et al (2011). Recent studies have focused on the thermodynamic disequilibrium introduced by biological activity in the entropy budget of a planetary atmosphere (Kleidon 2010; Simoncini et al 2013; Krissansen-Totton et al 2016), on its gas-phase isotope ratios (e.g., Snellen et al 2014), or on the surface composition (e.g., mineral coverage, Hystad et al 2015), reflectivity (e.g., vegetation pigments, Seager et al 2005; Gargaud et al 2011). Seager and Bains (2015) provide a recent review of the exoplanetary biosignature literature.

The experience gained so far with exoplanet atmospheric research shows that a continued challenge is to break the degeneracies in composition and climate associated with retrieval of atmospheric spectra (Burrows 2014) and simulations of future observations of Earth-like planets point to the same conclusions (von Paris et al 2013). The emerging vision for identifying biosignatures by remote sensing is that observing and studying the atmospheres of a large number of exoplanets will be required to provide an essential context for interpreting detections of possible biosignatures and for determining the prevalence of life beyond Earth. A good approach could be to create a Hertzsprung-Russell diagram for planetary atmospheres and species suggestive of life. Only after observing a large number of planetary atmospheres, will it be possible to identify an anomaly in this diagram that cannot be explained without the presence of life.

\subsection{The need for direct imaging in the mid-infrared}

In order to better understand planetary atmospheres and to provide an essential context for interpreting detections of possible biosignatures, direct imaging in the mid-infrared has a key role to play. In particular, this wavelength regime is ideal to infer the presence of an atmosphere and basic planetary properties, to measure the atmospheric composition of important species such as $\mathrm{H}_{2} \mathrm{O}, \mathrm{CO}_{2}, \mathrm{O}_{3}$, and $\mathrm{CH}_{4}$, and to understand the surface conditions and habitability of the planets. These points are discussed separately in the following sub-sections.

\subsubsection{Presence of an atmosphere and basic planetary properties}

For any atmospheric composition, monitoring the variations of thermal emission of an exoplanet during its orbital motion provides a fundamental diagnostic on the presence of a dense atmosphere and the nature of the climate (e.g., Selsis 2004). This is illustrated in Figure 0.2, which shows modeled examples of such orbital photometry for Earth analogs and three different mid-infrared wavelengths. With an increasing number of thermal emission bands monitored, more planet properties can be inferred or constrained by orbital photometry: rotation, albedo, obliquity, radius (Selsis et al 2011; Cowan et al 2012; Maurin et al 2012; Selsis et al 2013), presence of a large satellite (Moskovitz et al 2009), response to eccentricity (Bolmont et al 2016). Thermal phase curves have successfully been used to characterize unresolved transiting (Knutson et al 2007) and even non-transiting systems (Crossfield et al 2010) but the stellar variability and the required photometric precision make these measurements very difficult. With imaged planets this method will show its full potential. Although the inner working angle will limit the access to the smallest phase angles, only a moderate photometric precision of $\sim 10 \%$ will be required to achieve a crucial diagnostic and first classification of the targets. Another strength of the method comes from the favorable distribution of orbit orientations. For the median inclination of randomly oriented orbits $\left(60^{\circ}\right)$, the amplitude of phase curves is only decreased by $10 \%$ compared with the maximum variations, reached for a $90^{\circ}$ inclination (Maurin et al 2012). Only a few observations covering one orbit would be sufficient to start deriving constraints on the climate and several low-resolution or broadband observations will anyway be necessary to resolve the orbital motion of the planet. 


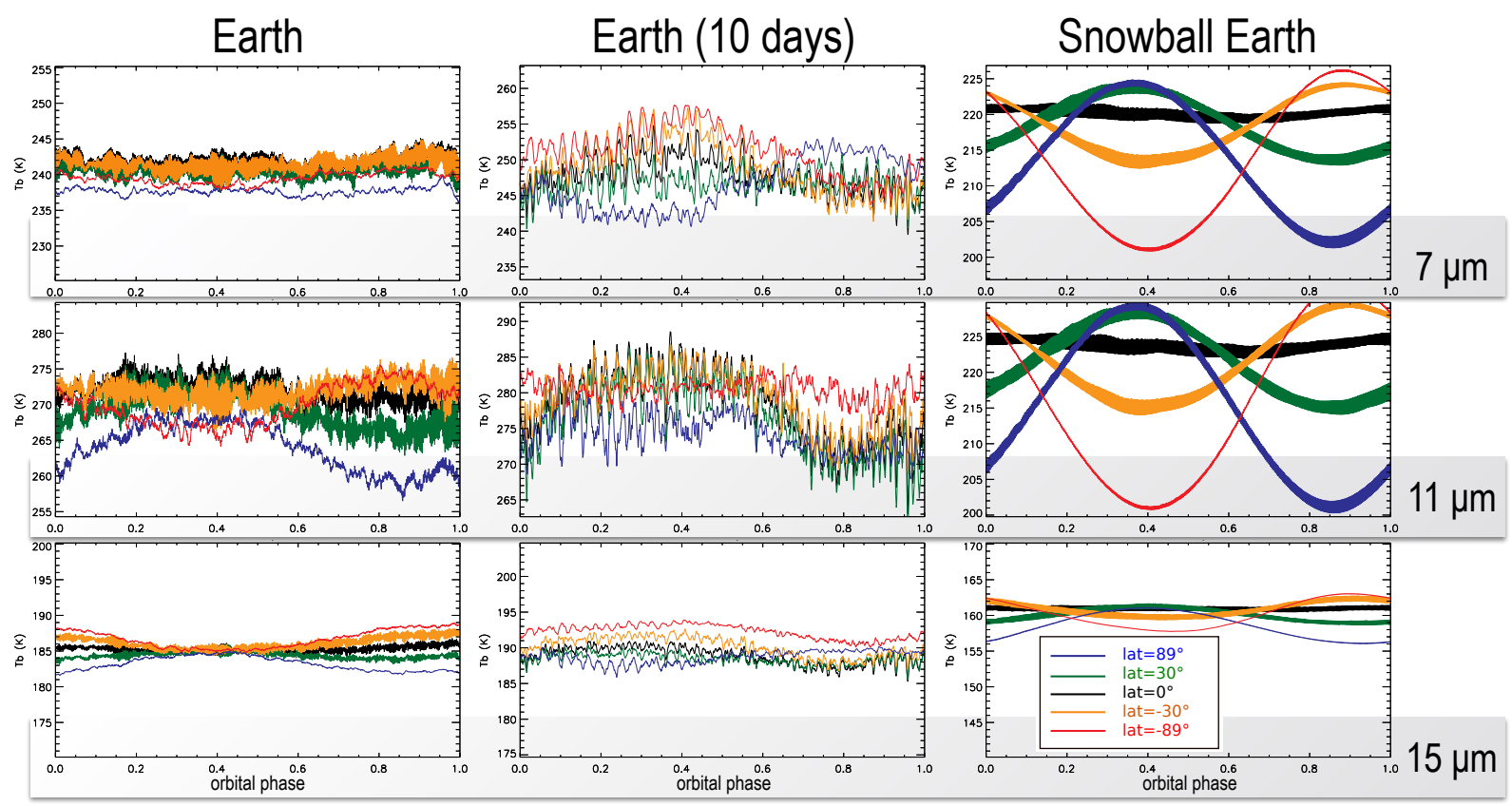

Fig. 0.2: Variability of the observed thermal emission from an Earth-like planet over one orbit (Gómez-Leal 2013). These lightcurves were simulated for three different planets: the Earth, an Earth analog rotating in 10 days, and a snowball Earth. They are given for different observer latitudes and 3 different $1 \mu \mathrm{m}$-wide bands: $7.1 \mu \mathrm{m}$ in a $\mathrm{H}_{2} \mathrm{O}$ band and probing the upper troposphere (or the surface in the snowball case), $11 \mu \mathrm{m}$ in an atmospheric window and probing the surface boundary layer or clouds, and $15.5 \mu \mathrm{m}$ in the $\mathrm{CO}_{2}$ band and probing the stratosphere. Flux is given as a brightness temperature (note the different scales).

Table 0.1: Information on planets that can be obtained from low-resolution $(\mathrm{R}<20)$ mid-infrared observations $(6-20 \mu \mathrm{m})$. Two continuum (Cont.) bands ( 2 and 3 ) are also given, where in a cloud-free atmosphere, emission from the surface might be seen. The existence of an atmosphere can also be determined via spectral light curves.

\begin{tabular}{|c|c|c|c|c|c|}
\hline Information on planet & Species & IIN $[\mu \mathrm{m}$ & $\lambda_{\mathrm{MAX}}[\mu \mathrm{m}$ & $\overline{\mathrm{AVG}}[\mu \mathrm{m}]$ & $\bar{R}$ \\
\hline 1 Orbit characteristics & Cont. 1 & 6.00 & 20.0 & 13.0 & 1 \\
\hline 2 Combination of temperature, & Cont. 2 & 10.1 & 12.4 & 11.2 & 5 \\
\hline radius, and albedo & Cont. 3 & 8.16 & 9.24 & 8.67 & 8 \\
\hline 3 Existence of atmosphere & Cont. 1 & 6.00 & 20.0 & 13.0 & 1 \\
\hline & $\mathrm{CO}_{2}$ & 9.07 & 9.56 & 9.31 & 19 \\
\hline & & 10.1 & 10.7 & 10.4 & 16 \\
\hline & & 13.3 & 17.0 & 15.0 & 4 \\
\hline 4 Presence of water & $\mathrm{H}_{2} \mathrm{O}$ & 6.67 & 7.37 & 7.00 & 10 \\
\hline & & 17.4 & 25.0 & 20.5 & 3 \\
\hline 5 Suggestion of life & $\mathrm{CH}_{4}$ & 7.37 & 7.96 & 7.65 & 13 \\
\hline & & 7.37 & 8.70 & 7.98 & 6 \\
\hline & $\mathrm{O}_{3}$ & 9.37 & 9.95 & 9.65 & 17 \\
\hline
\end{tabular}

\subsubsection{Atmospheric composition}

In addition to the constraints from orbital broadband photometry, mid-infrared spectroscopy is fundamental to investigate the nature of planetary atmospheres by detecting spectral features, constraining the temperature and pressure structure, the cloudiness and by determining whether the planet could potentially harbor life. Indeed, several important species have mid-infrared spectral signatures that can be detected at low to medium spectral resolving power (e.g., 
$\mathrm{H}_{2} \mathrm{O}, \mathrm{CO}_{2}, \mathrm{O}_{3}, \mathrm{CH}_{4}$, see Table 0.1 ). To illustrate how the properties of an exoplanetary atmosphere are imprinted in the mid-infrared spectrum, we show in Figure 0.3 eight examples of $\mathrm{HZ}$ rocky planets with various atmospheres and orbiting two different nearby stars (i.e., Proxima Cen and $\varepsilon$ Eri). For Proxima Cen (M6V star), we use the actual properties of the recently-announced planet (mass and distance to the star, Anglada-Escudé et al 2016) to compute four different examples of planet composition (Cases 1 to 4): a stripped planet $\left(\mathrm{M}=4.0 \mathrm{M}_{\oplus}, \mathrm{R}=1.5 \mathrm{R}_{\oplus}\right.$ ) without any atmosphere (Case 1), a water ocean planet $\left(\mathrm{M}=2.0 \mathrm{M}_{\oplus}, \mathrm{R}=1.5 \mathrm{R}_{\oplus}\right.$, Léger et al 2004), and two cases of large rocky planets: one with a strong $\mathrm{CO}_{2}$ Greenhouse effect $\left(\mathrm{P}_{\mathrm{CO} 2}=300 \mathrm{mbar}\right)$ and possibly biotic $\mathrm{O}_{2}$ (Case 3) and one without any $\mathrm{O}_{2}$ input (Case 3.5). For $\varepsilon$ Eri, Case 4 shows a mini-neptune $\left(\mathrm{M}=4.0 \mathrm{M}_{\oplus}, \mathrm{R}=1.8 \mathrm{R}_{\oplus}\right)$ in the HZ, Case 5 shows a waterocean planet $\left(M=2.0 \mathrm{M}_{\oplus}, \mathrm{R}=1.5 \mathrm{R}_{\oplus}\right)$ in the $\mathrm{HZ}$, Case 6 shows a large Earth-analog planet $\left(\mathrm{M}=4.0 \mathrm{M}_{\oplus}, \mathrm{R}=1.5 \mathrm{R}_{\oplus}\right)$, and Case 6.5 a large Earth-analog planet as in case 6, but without $\mathrm{O}_{2}$ input as in case 4. While these eight examples likely represent only a tiny fraction of the possible cases we might encounter in Nature, they illustrate the wealth of information contained in the mid-infrared regime.

\subsubsection{Surface conditions and habitability}

The surface temperature (defined to be that at the interface between the solid/liquid surface and any atmosphere) is a key property to study the habitability of an exoplanet and to search for life. It is different from the effective temperature (i.e., that of a blackbody having the same surface area and the same insolation), which can be computed from the stellar luminosity and hence orbital parameters obtained by indirect detection techniques. Measuring the surface temperature of an exoplanet requires to detect its photon and, here again, the mid-infrared regime is crucial to achieve this goal. For planets similar to Earth, a detailed study by von Paris et al (2013) shows that surface conditions (temperature and pressure) can be characterised relatively well from mid-infrared observations (to within $\sim 10 \mathrm{~K}$ at 3- $\sigma$ ) with $\mathrm{S} / \mathrm{Ns}$ between 10 and 30, depending on spectral resolution. By observing a large number of HZ rocky exoplanets, it will be possible to correlate the concept of habitability with key parameters and processes like spectral type of the parent star, degree of stellar activity, the temperature/pressure structure of the atmosphere, gaseous composition, the circulation and heat transfer of the atmosphere, the atmospheric chemistry and photochemistry, and the outgassing of atmospheric species.

\subsection{Measurement approach}

\subsubsection{Observing challenge}

To study the atmospheres of exoplanets, a key requirement is to obtain high-quality spectra over a broad range of wavelengths. This is a very challenging observational task due to several factors and particularly the high contrast and the small angular separation between the planet and its host star. Figure 0.4 (left) shows the contrast at $10 \mu \mathrm{m}$ between a $300 \mathrm{~K}$ Earth-sized planet and a sample of nearby single main sequence stars (Kaltenegger et al 2010) as a function of the angular separation between the middle of the habitable zone around these stars and the star itself. The contrast ranges between a few $10^{-8}$ for F-type stars to almost $10^{-4}$ for late-type $\mathrm{M}$ stars, while the angular separation ranges between a few mas for the furthest $\mathrm{M}$ stars to 200 mas for the closest $\mathrm{F}$ stars. In order to spatially resolve the closest systems (within $10 \mathrm{pc}$ ) in the mid-infrared, an aperture at least $80 \mathrm{~m}$ in diameter would be required. A monolithic telescope of this size is presently not feasible, especially since the observatory must be space-borne and cooled to avoid the thermal background from the Earth atmosphere and provide continuous coverage and sensitivity between 6 and $20 \mu \mathrm{m}$.

Another major observing challenge comes from the low flux received from distant $300 \mathrm{~K}$ Earth-sized planets. This puts strong requirements on the instrumental sensitivity and has a huge impact on the number of planets that can be studied during a given mission lifetime. To illustrate this issue, the right part of Figure 0.4 shows the flux at $10 \mu \mathrm{m}$ as a function of angular separation of putative $300 \mathrm{~K}$ blackbody planets located in the middle of the $\mathrm{HZ}$ of nearby single main-sequence stars. The $10-\sigma$ sensitivity in one day of current and planned mid-infrared instruments is indicated by the colored rectangles. They show that no current or planned mid-infrared instruments have both the sensitivity 


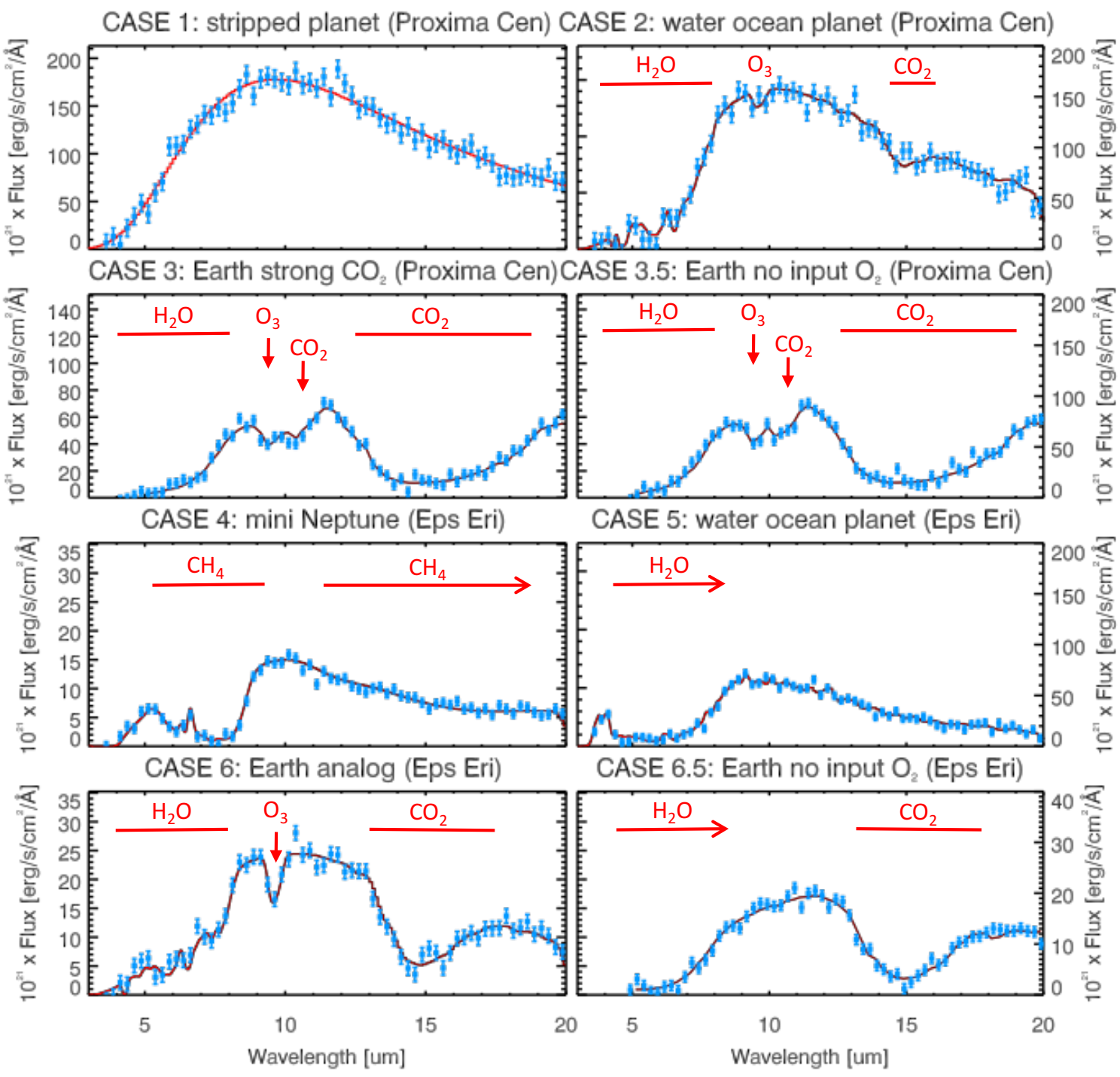

Fig. 0.3: Simulated mid-infrared spectra of planets with various atmospheric properties that can be studied by remote sensing (red line). The synthetic spectra are computed by coupled climate chemistry models (Cases 1, 2, 4, 5, Rauer et al. 2011; Cases 3, 3.5, 6, 6.5, Tian et al. 2014) for planets around Proxima Cen (M6V) and $\varepsilon$ Eri (K2V). Overplotted in blue are simulated observations (R=40), imposing a $\mathrm{S} / \mathrm{N}$ of 20 on continuum detection at $10 \mu \mathrm{m}$. Besides $\mathrm{O}_{3}$ in the atmosphere of the water ocean planet around Proxima Cen (Case 2), all spectral features can be retrieved in a single visit with these requirements $(\mathrm{R}=40$ and $\mathrm{S} / \mathrm{N}=20)$. Detecting $\mathrm{O}_{3}$ in Case 2 would require a higher $\mathrm{S} / \mathrm{N}$ or follow-up observations. See main text for more information.

and the angular resolution required to observe a significant number of systems. On one hand, ELT-like ground-based telescopes will have the required angular resolution but a limited sensitivity. On the other hand, a space-based telescope such as JWST/MIRI is planned to reach the required sensitivity but will not provide the necessary angular resolution to resolve even the closest systems. Let's note nonetheless that exciting perspectives exist today on a limited number of stars. For instance, VLT/VISIR has the necessary angular resolution and sensitivity to image in approximately 100 hours a super-Earth $\left(\sim 2 R_{\oplus}\right)$ exoplanet around our nearest $G$ star (i.e., $\alpha$ Cen) and will be upgraded soon to achieve this objective. The future mid-infrared imager METIS on the ELT might also detect $\sim 10$ small planets (1 to $4 \mathrm{R}_{\oplus}$ ) with equilibrium temperatures between 200 and $500 \mathrm{~K}$ around the nearest stars (Quanz et al 2015). 

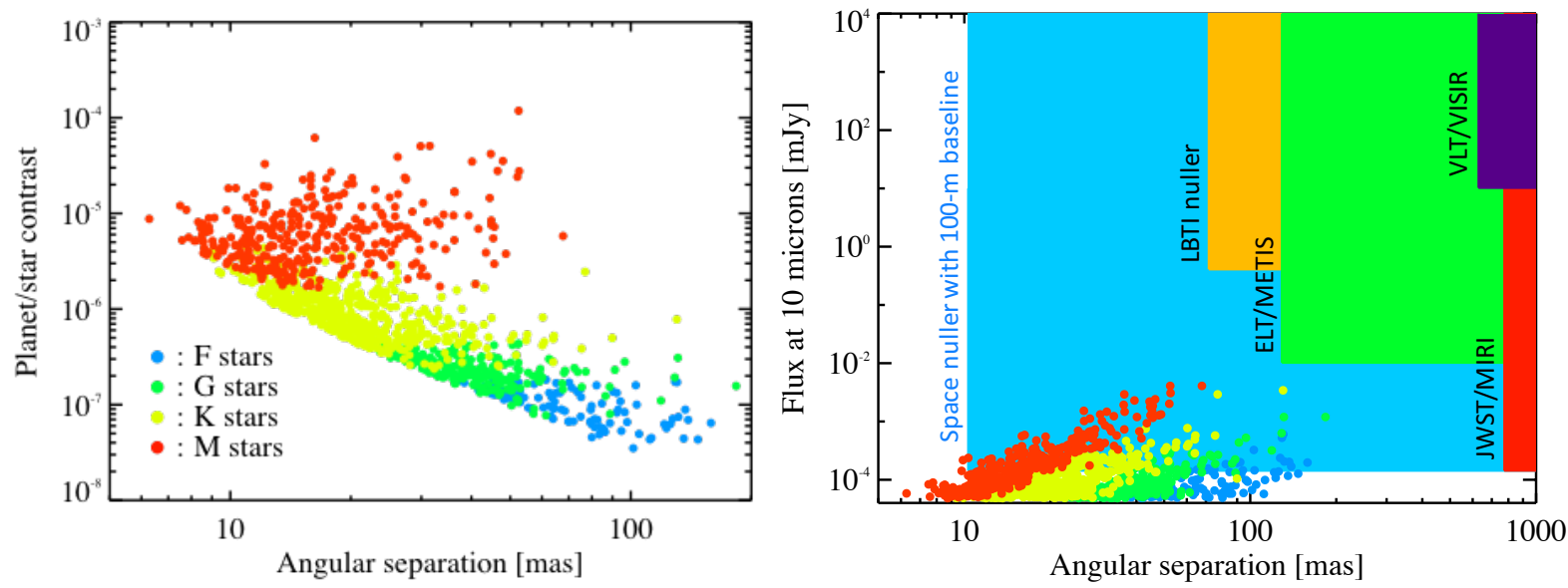

Fig. 0.4: Left, planet/star contrast as a function of angular separation for putative $300 \mathrm{~K}$ rocky planets around nearby single mainsequence stars (stellar sample from Kaltenegger et al 2010). Right, corresponding flux at $10 \mu \mathrm{m}$ of these planets and 10- $\sigma$ sensitivity in one day of current and planned mid-infrared instruments (see colored rectangles, values scaled from Brandl et al 2014). The sensitivity and angular resolution of a four $2 \mathrm{~m}$-aperture space-based nulling instrument are represented by the blue rectangle. A space-based nulling interferometer would achieve a similar sensitivity to JWST but would provide an increase of angular resolution by almost two orders of magnitudes (for a baseline length of $100 \mathrm{~m}$ ), which would allow a large number of planetary atmospheres to be studied.

\subsubsection{Extracting the planetary photons by nulling interferometry}

Nulling interferometry is a technique initially proposed in 1978 to detect "non-solar" planets (Bracewell 1978). The basic principle of this technique is to combine the beams coming from two telescopes in phase opposition so that a dark fringe appears on the line of sight (see middle panel of Figure 0.5), which strongly reduces the stellar emission. On the other hand, off-axis emission, such as that of a planet, can be transmitted by optimizing the baseline length. This technique is very promising to fulfill all the requirements described in the previous section because the distance between the telescopes can be adjusted to provide the necessary angular resolution to resolve the exoplanet from its host star while stellar photons can be removed from the measured spectrum to maximize contrast and sensitivity. However, even when the stellar emission is sufficiently reduced, it is generally not possible to detect Earth-like planets with a static array configuration, because their emission is dominated by the thermal contribution of a series of other extraneous and generally dominant signals originating from the telescope itself (thermal background, readout noise), material in the Solar system (the local zodiacal emission), or around the target star (exozodiacal light). This is the reason why Bracewell proposed to rotate the interferometer so that the planetary signal is modulated by alternatively crossing high and low transmission regions, while the stellar signal and the background emission remain constant. The planetary signal can then be retrieved by synchronous demodulation. This modulation technique is in many ways similar to the use of a chopper wheel that allows the detection of infrared sources against a thermal background and/or drifting detector offsets.

\subsubsection{The Emma X-array configuration}

During the DARWIN/TPF-I studies, it was quickly realized that the interferometric array cannot be rotated sufficiently fast to mitigate low frequency instrumental drifts to a level sufficiently low to enable the observation of Earth-like exoplanet (e.g., Lay 2004). A solution proposed to overcome this problem is to use more than two telescopes and phase chopping, which consists in synthesizing two different transmission maps with the same telescope array, by applying different phase shifts in the beam combination process (see Figure 0.5). In addition to allowing more precise differential measurements, it is also possible to isolate the planetary signal from the contributions of symmetric brightness emissions such as the star, local zodiacal cloud, exozodiacal cloud, stray light, thermal, or detector gain. After the investigation of several interferometer architectures (e.g., Angel et al 1997; Mennesson and Mariotti 1997; Blackwood et al 2003), two array architectures have been thoroughly investigated by ESA during two parallel assess- 


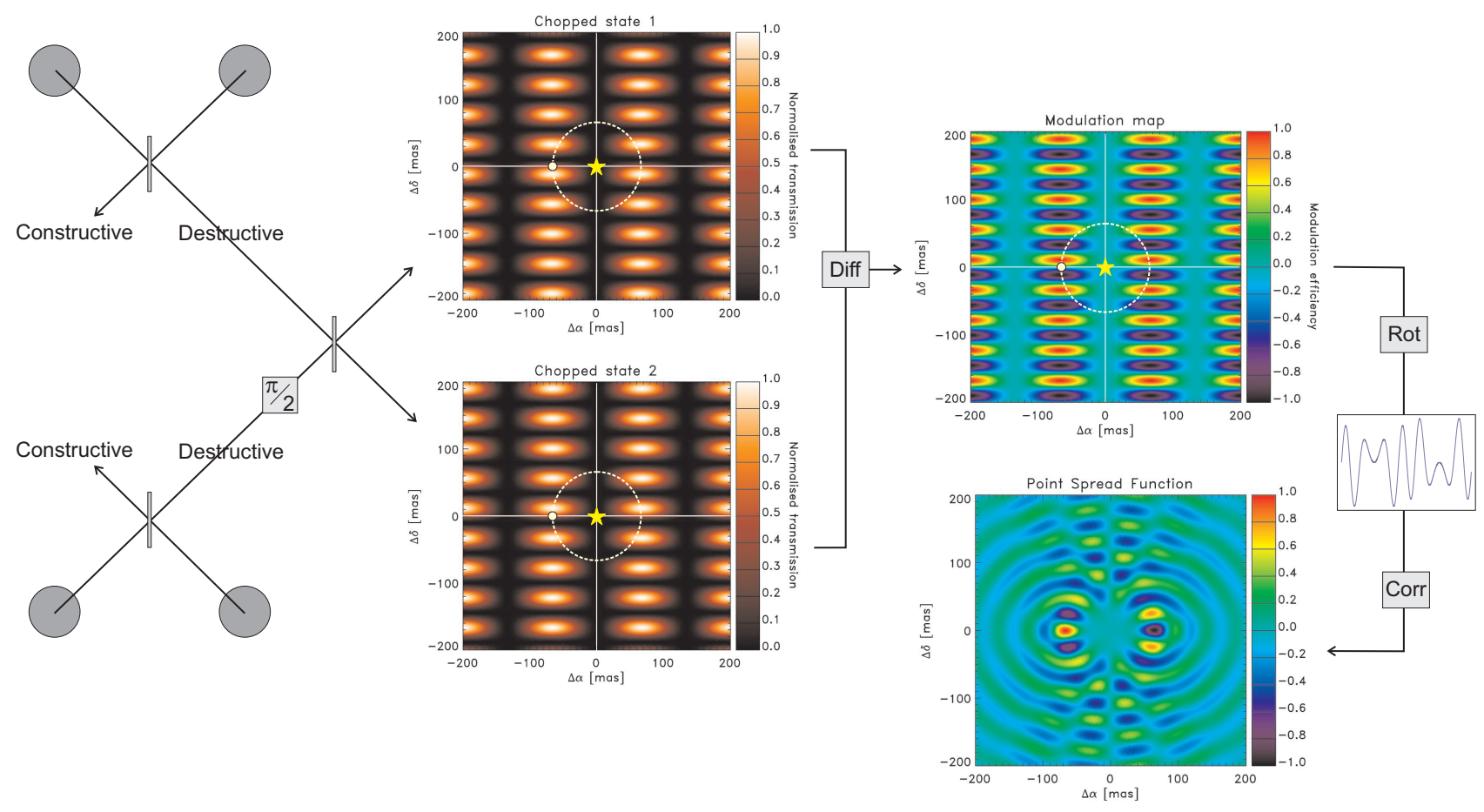

Fig. 0.5: Overview of phase chopping for the $X$-array configuration. Combining the beams with different phases produces two conjugated transmission maps (or chop states), which are used to produce the chopped response. Array rotation then locates the planet by cross-correlation of the modulated chopped signal with a template function (figure from Cockell et al 2009).

ment studies carried out by EADS Astrium and Alcatel-Alenia Space in 2005-2006: the four-telescope X-array and the Three-Telescope Nuller (TTN, Karlsson et al 2004). These studies included the launch requirements, payload spacecraft, and the ground segment during which the actual mission science would be executed. Almost simultaneously, NASA/JPL initiated a similar study for TPF-I and focused in particular on the Dual-chopped Bracewell (Velusamy et al 2003; Lay 2004) and X-array configurations. These efforts on both sides of the Atlantic have finally resulted in a convergence and consensus on mission architecture, the so-called non-coplanar (aka Emma-type) X-array (represented in Figure 0.6). The baseline design consisted in four 2-m aperture collector spacecraft, flying in rectangular formation and feeding light to the beam combiner spacecraft located approximately $1200 \mathrm{~m}$ above the array. This arrangement makes available baselines up to $170 \mathrm{~m}$ for nulling measurements and up to $500 \mathrm{~m}$ for the general astrophysics program (constructive imaging).

The number of planetary atmospheres that can be studied by such an instrument is a critical metric to estimate the science return of the mission. This number depends on a series of astrophysical and instrumental parameters. For this exercise, we will assume that each nearby star has one HZ rocky planet and that the exozodiacal dust disks are no brighter than the solar zodiacal cloud. Since it would be impossible to cover all possible atmospheric compositions, we assume that each planet is a $300 \mathrm{~K}$ blackbody and require an $\mathrm{S} / \mathrm{N}$ of at least 20 on the detection of the continuum with a spectral resolution of 40 . We consider a four telescope Emma X-array interferometer as emerged from the DARWIN/TPF studies 10 years ago (Cockell et al 2009). The simulated mission performances are computed using the DARWINSim simulator (Defrère et al 2010) that has been extensively validated and cross checked with a similar tool developed independently at NASA/JPL for the TPF-I mission (Lay et al 2007; Defrère et al 2008b). The results of the simulations are shown in Table 0.2 for two different planet radii (i.e., $1.0 \mathrm{R}_{\oplus}$ and $1.5 \mathrm{R}_{\oplus}$ ). Over a 3-year mission lifetime, a nulling interferometer with $0.75-\mathrm{m}$ (resp. 2-m) apertures could characterize the atmospheres of 12 to 27 (resp. 32 to 71) $\mathrm{HZ}$ planets, depending on their size (assuming a spectral resolution of 40). Additional mission lifetime could be spent on follow-up observations to study in more details the most interesting planets and/or on a detection phase if the planets are not known in advance. Note that a Bracewell interferometer (two telescopes) would be another possible option for a mission focused only on M and K stars (Defrère 2009). 


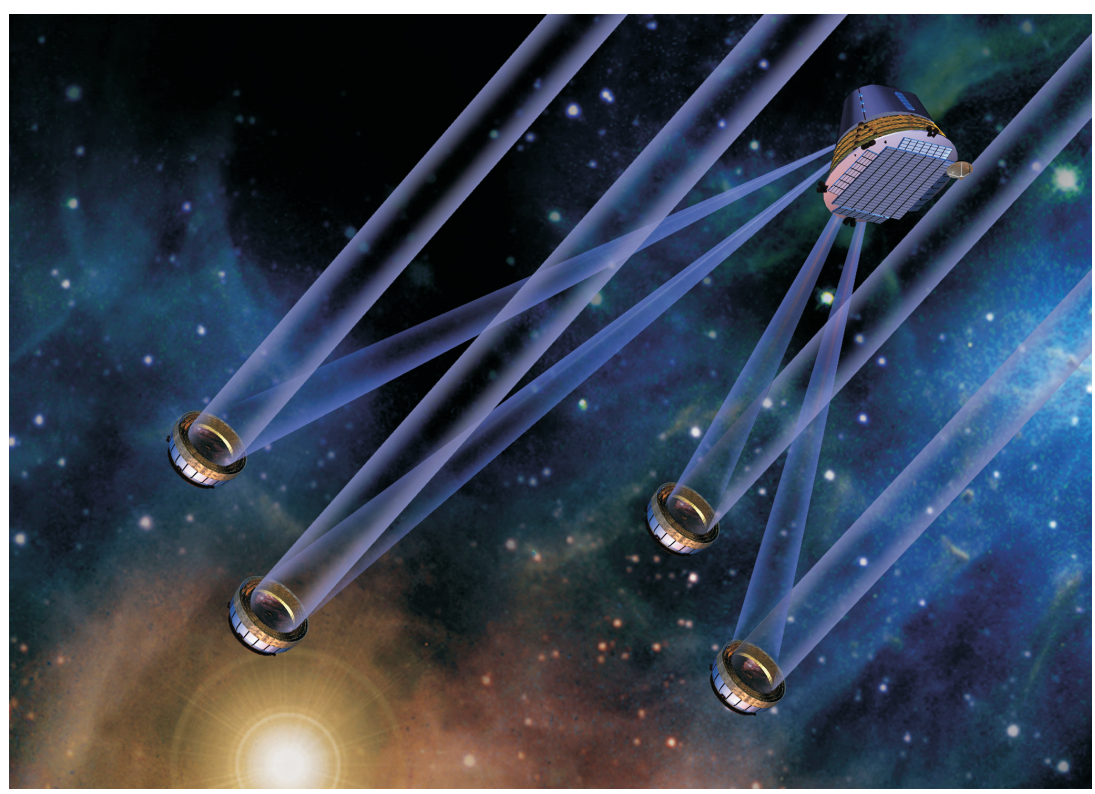

Fig. 0.6: Artist impression of the DARWIN/TPF space interferometer in its "Emma X-array" baseline configuration. It presents four telescopes and a beam combiner spacecraft, deployed and observing at the Sun-Earth Lagrange point L2.

Table 0.2: Number of rocky planet atmospheres that can be studied over a 3-year mission by an Emma X-array nulling interferometer (four apertures), assuming that each nearby main-sequence star has a $\mathrm{HZ}$ exoplanet (with an equilibrium temperature of $300 \mathrm{~K}$ ). Additional mission lifetime could be spent on follow up observations to study in more details the most interesting planets and/or a detection phase if the planets are not known in advance. Note that a Bracewell configuration (two apertures) is another possible option for a mission focused on $\mathrm{M}$ and $\mathrm{K}$ stars.

\begin{tabular}{|c|c|c|c|c|c|c|}
\hline$\overline{\text { Configuration }}$ & Tel. diame & $\overline{\mathrm{Pl} \text { Pl. radiu }}$ & $\mathrm{M}$ & $\overline{\mathrm{KC}}$ & $\mathrm{F}$ & \\
\hline \multirow{4}{*}{ Emma X-array (4T) } & $0.75 \mathrm{~m}$ & $1.0 \mathrm{R}_{\oplus}$ & 8 & 31 & 0 & 12 \\
\hline & & $1.5 \mathrm{R}_{\oplus}$ & 18 & 62 & & 27 \\
\hline & $2.0 \mathrm{~m}$ & $1.0 \mathrm{R}_{\oplus}$ & & 86 & & 32 \\
\hline & & $1.5 \mathrm{R}_{\oplus}$ & & & 23 & 71 \\
\hline
\end{tabular}

\subsubsection{State-of-the-art}

\subsubsection{Formation flying}

Formation flying is a key technology for the deployment and success of a space-based interferometer. Remarkable advances in technology have been made in Europe in recent years with the space-based demonstration of this technology by the PRISMA mission (http://www.snsb.se/en/Home/Space-Activities-in-Sweden/ Satellites/and http://www.ohb-sweden.se/space-missions/prisma/). PRISMA demonstrated a sub-cm positioning accuracy between two spacecraft, mainly limited by the metrology system (GPS and RF). The launch of ESA's PROBA-3 mission in 2018 will provide further valuable free-flyer positioning accuracy results (sub$\mathrm{mm}$ ), which exceeds the requirements for a space-based interferometer. Extending the flight-tested building-block functionality from a distributed two-spacecraft instrument to an instrument with more spacecraft mainly relies on the replication of the coordination functionality and does not present additional complexity in terms of procedures according to the PRISMA navigation team. While formation flying can then be considered to have reached TRL 9 once PROBA-3 has flown, an uncertainty remains regarding fuel usage and the possible lifetime of such a mission. 


\subsubsection{Spatial filters}

Spatial filters are an important technology for nulling interferometry because they significantly reduce the optical aberrations in wavefronts, making extremely deep nulls possible. To provide spatial filtering over large bandwidths, a variety of ways can be used, including single-mode fiber optics, photonic crystal fibers, or integrated optics. Developments of single-mode fibers for the mid-infrared were funded by NASA between 2003 and 2008 (Ksendzov et al 2007, 2008). Fiber optics made of chalcogenide and silver halide materials have been demonstrated to yield $25 \mathrm{~dB}$ or more rejection of higher-order spatial modes at $10 \mu \mathrm{m}$, but they would require to divide the 6-20 $\mu \mathrm{m}$ band into two parts. Although this performance is sufficient for flight, it would be greatly advantageous to improve the throughput of these devices and to test them over the full wavelength range in cryogenic conditions. Spatial filter technology would then be at TRL 5. The spatial filtering capabilities of photonic crystal fibers should also be investigated for use at mid-infrared wavelengths, because of the improved throughput that they may provide and the possibility to cover the whole wavelength range with a single technology. Note finally that, with recent developments in wavefront control with extreme adaptive optics systems (Jovanovic et al 2015, e.g.,), it is not clear whether such a technology will be required. This should be addressed in the future.

\subsubsection{Beam combination}

Classical optics designs of four-telescope nulling beam combiners (Martin and Booth 2010) have been demonstrated to flight requirement levels but at room temperature and using signals much stronger than the astronomical ones. Recently, a very promising grating nuller approach was proposed and shown to achieve nulls of $4 \times 10^{-5}$ over the full $18 \%$ bandwidth K-band (Martin et al 2017). Alternatively, integrated optics (IO) beam combination can be achieved by a network of single-mode waveguides embedded in a cm-scale glass chip. This solution bypasses complex optical interferometric trains sensitive to vibrational, thermal and mechanical stress, hence reducing the risk associated with bulky science instruments. As fibers do, IO can furthermore achieve wavefront cleaning to mitigate phase errors (Wallner et al 2002; Mennesson et al 2002). Silica-based IO solutions are now being successfully implemented in operating near-infrared 4-telescope interferometers (Eisenhauer et al 2011; Le Bouquin et al 2011) and have also been investigated for nulling applications in the near-infrared at $1.5 \mu \mathrm{m}$ (Weber et al 2004; Errmann et al 2015) with stable nulls down to $10^{-4}$ over $5 \%$ bandwidth. Because silica glasses are opaque to IR radiation for $\lambda>2 \mu \mathrm{m}$, the extension of the IO approach to the mid-infrared in the 3-30 $\mu \mathrm{m}$ range requires an adequate material and technological platform to manufacture high quality optical chips.

\subsubsection{Starlight suppression}

A considerable expertise has been developed on starlight suppression over the past 20 years, both in academic and industrial centers accross the globe. Approximately $35 \mathrm{PhD}$ theses were dedicated to this topic and more than 40 refereed papers. These efforts culminated with laboratory demonstrations at room temperature mainly at the Jet Propulsion Laboratory (JPL) in the US. For instance, work with the Adaptive Nuller has indicated that mid-infrared nulls of $10^{-5}$ are achievable with a bandwidth of $34 \%$ and a mean wavelength of $10 \mu \mathrm{m}$ (Peters et al 2010). Another testbed, the planet detection testbed, was developed in parallel and demonstrated the main components of a high performance four-beam nulling interferometer at a level matching that needed for the space mission (see Figure 0.7). At $10 \mu \mathrm{m}$ with $10 \%$ bandwidth, it has achieved nulling of $8 \times 10^{-6}$ (the flight requirement is $10^{-5}$ ), starlight suppression of $10^{-8}$ after post-processing, and actual planet detection at a planet-to-star contrast of $3 \times 10^{-7}$, i.e., the Earth-Sun contrast, but with fluxes much higher than those expected from stars and planets allowing working at room temperature without being disturbed but the thermal emission of the environment. The phase chopping technique (Mennesson et al 2005) has also been implemented and validated on-sky with the Keck Nuller Interferometer (Colavita et al 2009). A null stability of a few $\sim 10^{-3}$ was achieved, mainly limited by the large thermal background and variable water vapor content, both effects specific to ground based mid-infrared observations. The next step would be to reproduce this experiment in cryogenic conditions and would require the successful validation of cryogenic spatial filters and the implementation of a cryogenic deformable mirror, which is now within reach (Enya et al 2009). 


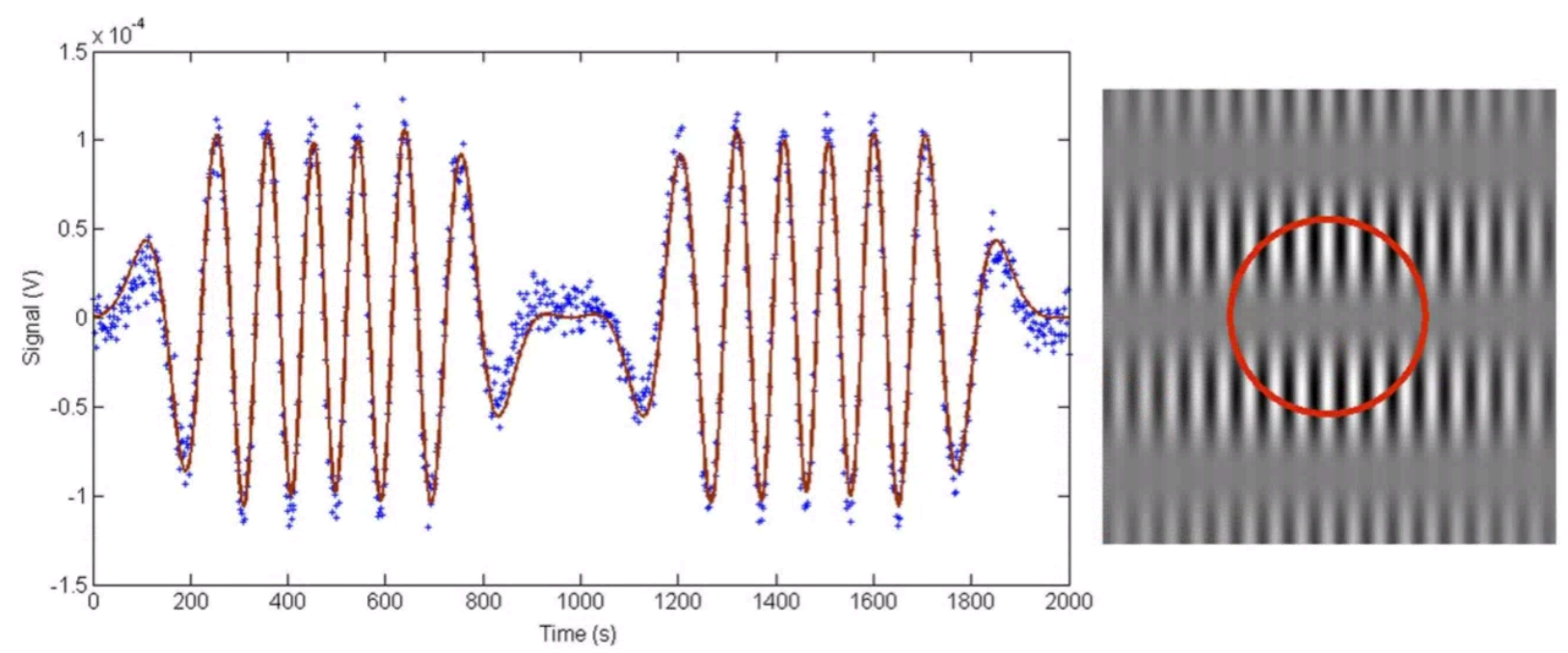

Fig. 0.7: Planet signal detected with the Planet Detection Testbed (Martin et al 2012). Markers indicate planet signal from nulling detector with star shuttered off. On this scale, the star would be $250 \mathrm{~V}$. Each point is an item of data from the 2-s chop cycle and the whole trace shows the null signal obtained over a 360 degree effective rotation of the interferometer array. The line is a fit to the signal from a planet at a nominal angular radius of $6.35 \times 10^{-7}$ rad (or 132 mas) from the star. By comparison, the equivalent angular fringe distance from the short baseline is $4.7 \times 10^{-7} \mathrm{rad}$. Near the center and at the ends of the plot, the planet crosses the null fringe. Right: equivalent sensitivity map of the interferometer array. Array rotation causes the planet location to orbit (solid line) around the central null fringe (gray), and thus its signal is modulated both by the higher frequency fringes on the long baseline and by the chopping.

In parallel, the operation of high-precision ground-based interferometers has matured in both Europe and the US. In particular, Europe has gained a strong expertise in the field of fringe sensing, tracking, and stabilization with the operation of the Very Large Telescope Interferometer (VLTI). In the United states, a lot of technical expertise was gained by operating several nulling interferometers such as the Keck Interferometer Nuller (KIN, Colavita et al 2009), the Palomar Fiber Nuller (PFN, Mennesson et al 2011b), and the Large Binocular Telescope Interferometer (Hinz et al 2014). All have produced excellent scientific results (e.g., Mennesson et al 2014; Defrère et al 2015) and pushed high-resolution mid-infrared imaging to new limits (Defrère et al 2016). New innovative data reduction techniques have also been developed to improve the accuracy of nulling instruments (Hanot et al 2011; Mennesson et al 2011a) but more work is required to adapt this technique to four-telescope configurations.

\subsection{Prospects}

\subsubsection{Current context}

Interferometric telescope arrays operating at infrared wavelengths provide already exciting science at ground-based facilities, such as ESO's VLTI and the US CHARA, LBT, and NPOI facilities. This leads to opportunities for testing technologies on the ground and to push new developments ahead in collaboration with the existing interferometry community. Within this community, there is currently a science-driven, international initiative to develop the roadmap for a future ground-based facility that will be optimised to image planet-forming disks on the spatial scale where the protoplanets are assembled, which is the Hill Sphere of the forming planets. This Planet Formation Imager (PFI, Monnier et al 2016) shall detect and characterise protoplanets during their first $\sim 100$ million years and trace how the planet population changes due to migration processes, unveiling the processes that determine the final architecture of exoplanetary systems. With $\sim 20$ telescope elements and baselines of $\sim 3 \mathrm{~km}$, the PFI concept is optimised for imaging complex scenes at mid-infrared wavelengths $(3-12 \mu \mathrm{m})$ and at 0.1 milliarcsecond resolution, complementing the capabilities of a space interferometer that would be optimised to achieve the sensitivity and contrast required to characterise the atmospheres of mature exoplanets. A space-based interferometer and PFI will share many common technology challenges, for instance on mid-infrared beam combination and nulling schemes, and we are well posi- 
tioned to exploit synergies resulting between these projects.

Regarding space agencies, NASA is currently exclusively focused on visible (and possibly near infrared) wavelengths for future exoplanet missions (internal and external coronagraphs) such as the Habitable Exoplanet Imaging mission ([HabEx, Mennesson et al 2016) and the Large UltraViolet Optical Infrared mission (LUVOIR, Crooke et al 2016) currently studied in preparation for the 2020 US Decadal Survey in Astronomy. Although NASA's consideration of flagship missions capable of detecting and characterising Earth-like planets is focused solely on direct imaging with large single apertures, there are opportunities for smaller-scale missions design to use other techniques (interferometry, astrometry, transit spectroscopy). In particular, interferometry has been identified as a key technique for future astrophysical observatories in the NASA Astrophysics Roadmap.

\subsubsection{Precursor concepts}

Several precursor instruments have been proposed and realized to prepare the way for a DARWIN/TPF-I mission. In particular, NASA funded two ground-based nulling interferometers (i.e., the KIN and the LBTI, see Section 0.5.4.4) for precursor science and technology demonstration. In Europe, a similar project was also seriously considered (GENIE, Fridlund and Gondoin 2003; Gondoin et al 2004). Regarding space-based precursor missions, there are the free-flying demonstrators, PRISMA and PROBA-3, already discussed in Section 0.5.4.1. In addition, concepts of small-scaled space-based infrared nulling interferometers were seriously considered both in Europe and in the US.

- FKSI (Fourier-Kelvin Stellar Interferometer, NASA) is a project consisting of a structurally connected infrared space interferometer with 0.5 -m diameter telescopes on a 12.5 - $\mathrm{m}$ baseline, passively cooled down to $60 \mathrm{~K}$ (Danchi et al 2008). The project was studied to the phase A level by the Goddard Space Flight Center in preparation for submission as a Discovery-class mission. With an angular resolution of 40 mas at a $5 \mu \mathrm{m}$ center wavelength, FKSI would exceed the angular resolution of JWST by a factor of 5. The main scientific goals would be the detection and characterization of extrasolar planets (including super-Earth around $\mathrm{M}$ stars), debris discs, and exozodiacal dust (e.g., Defrère et al 2008a).

- Pegase is a project consisting of a free-flying infrared space interferometer dedicated to hot Jupiters and exozodiacal disks (Ollivier et al 2009). It was initially proposed in the framework of the 2004 call for ideas by the French space agency (CNES) for its formation flying demonstrator mission. CNES performed a Phase 0 study in 2005 and concluded that the mission is feasible within an 8 to 9 years development plan, but the mission was not selected for budgetary reasons.

\subsubsection{Required technological developments}

The main development required to bring the technology of the proposed concept to TRL5 is the implementation of a cryogenic interferometer system that achieves the necessary starlight suppression and actual planet detection from 6 to $20 \mu \mathrm{m}$ with optical fluxes similar to the astronomical ones. To achieve this goal, preliminary system studies are required to (i) define the cryogenic design for passive cooling of the optics and active cooling of the detectors; (ii) characterize and optimize the vibrations of the interferometer in cryogenic conditions; and (iii) develop spatial filters (if needed) and beam combiners that can provide the necessary performance from 6 to $20 \mu$ m under cryogenic conditions. Specific developments in terms of fringe tracking (taking into account residual vibrations) and data reduction will undoubtedly be needed to reach the required level of performance in terms of starlight rejection. We also expect that dedicated developments will be required in the field of mid-infrared detectors, although the JWST legacy will be particularly useful in this context. 


\section{Cross-references}

- Direct Imaging as an Exoplanet Discovery Method

- Space Missions for Exoplanets: Overview and Introduction

- Future Space Missions for Exoplanet Science

- Spectroscopic Direct Detection of Exoplanets

- Mass-Radius Relations of Telluric Planets

- Characterization of Exoplanets: Observations and Modeling of Orbital Phase Curves

- The Exoplanet Atmospheres' "Spectral Zoo"

- Exoplanet Atmosphere Measurements from Direct Imaging (Ground and Space)

- Atmospheric Retrieval for Exoplanet Atmospheres

- Atmospheres as the Window Into Exoplanet Habitability and Life

- The Habitable Zone: The Climatic Limits of Habitability

- Detecting Habitability

- Atmospheric Biosignatures

- Detecting Biosignatures

- Earth: Atmospheric Evolution of a Habitable Planet

- Future Exoplanet Research: High Contrast Imaging Techniques

\section{Acknowledgements}

The authors thank A. Léger, M. Fridlund, and B. Mennesson for reading and commenting on the manuscript. The authors would also like to thank F. Selsis, H. Rauer, M. Godolt, A. Garcia Munoz, J.L. Grenfell and F. Tian for providing figures and/or running simulations for Proxima Cen b. Some of research described in this publication was carried out in part at the Jet Propulsion Laboratory, California Institute of Technology, under a contract with the National Aeronautics and Space Administration.

\section{Bibliography}

Angel JR, Burge JH, Woolf NJ (1997) Detection and spectroscopy of exo-planets like Earth. In: Ardeberg AL (ed) Optical Telescopes of Today and Tomorrow, Proceedings SPIE, vol 2871, pp 516-519, DOI 10.1117/12.269076

Angel JRP, Cheng AYS, Woolf NJ (1986) A space telescope for infrared spectroscopy of earth-like planets. Nature322:341-343, DOI 10.1038/322341a0

Anglada-Escudé G, Amado PJ, Barnes J, Berdiñas ZM, Butler RP, Coleman GAL, de la Cueva I, Dreizler S, Endl M, Giesers B, Jeffers SV, Jenkins JS, Jones HRA, Kiraga M, Kürster M, López-González MJ, Marvin CJ, Morales N, Morin J, Nelson RP, Ortiz J, Ofir A, Paardekooper SJ, Reiners A, Rodríguez E, Rodrî-guez-López C, Sarmiento LF, Strachan JP, Tsapras Y, Tuomi M, Zechmeister M (2016) A terrestrial planet candidate in a temperate orbit around proxima centauri. Nature 536(7617):437-440

Beichman C (2000) NASA's Terrestrial Planet Finder (TPF). In: Schürmann B (ed) Darwin and Astronomy : the Infrared Space Interferometer, ESA Special Publication, vol 451, p 239

Beichman C, Benneke B, Knutson H, Smith R, Lagage PO, Dressing C, Latham D, Lunine J, Birkmann S, Ferruit P, Giardino G, Kempton E, Carey S, Krick J, Deroo PD, Mandell A, Ressler ME, Shporer A, Swain M, Vasisht G, Ricker G, Bouwman J, Crossfield I, Greene T, Howell S, Christiansen J, Ciardi D, Clampin M, Greenhouse M, Sozzetti A, Goudfrooij P, Hines D, Keyes T, Lee J, McCullough P, Robberto M, Stansberry J, Valenti J, Rieke M, Rieke G, Fortney J, Bean J, Kreidberg L, Ehrenreich D, Deming D, Albert L, Doyon R, Sing D (2014) Observations of Transiting Exoplanets with the James Webb Space Telescope (JWST). PASP126:1134, DOI 10.1086/679566

Beichman CA, Woolf NJ, Lindensmith CA (1999) The Terrestrial Planet Finder (TPF) : a NASA Origins Program to search for habitable planets

Beichman CA, Fridlund M, Traub WA, Stapelfeldt KR, Quirrenbach A, Seager S (2007) Comparative Planetology and the Search for Life Beyond the Solar System. Protostars and Planets V pp 915-928, astro-ph/0601469 
Blackwood GH, Serabyn E, Dubovitsky S, Aung M, Gunter SM, Henry C (2003) System design and technology development for the Terrestrial Planet Finder infrared interferometer. In: Coulter DR (ed) Techniques and Instrumentation for Detection of Exoplanets, Proc. SPIE, vol 5170, pp 129-143, DOI 10.1117/12.521311

Bolmont E, Libert AS, Leconte J, Selsis F (2016) Habitability of planets on eccentric orbits: Limits of the mean flux approximation. A\&A591:A106, DOI 10.1051/0004-6361/201628073, 1604.06091

Bracewell RN (1978) Detecting nonsolar planets by spinning infrared interferometer. Nature274:780, DOI 10.1038/ 274780a0

Brandl BR, Feldt M, Glasse A, Guedel M, Heikamp S, Kenworthy M, Lenzen R, Meyer MR, Molster F, Paalvast S, Pantin EJ, Quanz SP, Schmalzl E, Stuik R, Venema L, Waelkens C (2014) METIS: the mid-infrared E-ELT imager and spectrograph. In: Ground-based and Airborne Instrumentation for Astronomy V, Proc. SPIE, vol 9147, p 914721, DOI 10.1117/12.2056468, 1409.3087

Burrows AS (2014) Highlights in the study of exoplanet atmospheres. Nature513:345-352, DOI 10.1038/nature13782, 1409.7320

Cockell CS, Léger A, Fridlund M, Herbst TM, Kaltenegger L, Absil O, Beichman C, Benz W, Blanc M, Brack A, Chelli A, Colangeli L, Cottin H, Coudé du Foresto V, Danchi WC, Defrère D, den Herder JW, Eiroa C, Greaves J, Henning T, Johnston KJ, Jones H, Labadie L, Lammer H, Launhardt R, Lawson P, Lay OP, LeDuigou JM, Liseau R, Malbet F, Martin SR, Mawet D, Mourard D, Moutou C, Mugnier LM, Ollivier M, Paresce F, Quirrenbach A, Rabbia YD, Raven JA, Rottgering HJA, Rouan D, Santos NC, Selsis F, Serabyn E, Shibai H, Tamura M, Thiébaut E, Westall F, White GJ (2009) Darwin-A Mission to Detect and Search for Life on Extrasolar Planets. Astrobiology 9:1-22, DOI 10.1089/ast.2007.0227, 0805.1873

Colavita MM, Serabyn E, Millan-Gabet R, Koresko CD, Akeson RL, Booth AJ, Mennesson BP, Ragland SD, Appleby EC, Berkey BC, Cooper A, Crawford SL, Creech-Eakman MJ, Dahl W, Felizardo C, Garcia-Gathright JI, Gathright JT, Herstein JS, Hovland EE, Hrynevych MA, Ligon ER, Medeiros DW, Moore JD, Morrison D, Paine CG, Palmer DL, Panteleeva T, Smith B, Swain MR, Smythe RF, Summers KR, Tsubota K, Tyau C, Vasisht G, Wetherell E, Wizinowich PL, Woillez JM (2009) Keck Interferometer Nuller Data Reduction and On-Sky Performance. PASP121:1120-1138, DOI 10.1086/606063

Cowan NB, Voigt A, Abbot DS (2012) Thermal Phases of Earth-like Planets: Estimating Thermal Inertia from Eccentricity, Obliquity, and Diurnal Forcing. ApJ757:80, DOI 10.1088/0004-637X/757/1/80, 1205.5034

Crooke JA, Roberge A, Domagal-Goldman SD, Mandell AM, Bolcar MR, Rioux NM, Perez MR, Smith EC (2016) Status and path forward for the large ultraviolet/optical/infrared surveyor (LUVOIR) mission concept study. In: Society of Photo-Optical Instrumentation Engineers (SPIE) Conference Series, Proceedings SPIE, vol 9904, p 99044R, DOI 10.1117/12.2233084

Crossfield IJM, Hansen BMS, Harrington J, Cho JYK, Deming D, Menou K, Seager S (2010) A New $24 \mu \mathrm{m}$ Phase Curve for $v$ Andromedae b. ApJ723:1436-1446, DOI 10.1088/0004-637X/723/2/1436, 1008.0393

Danchi WC, Barry RK, Lawson PR, Traub WA, Unwin S (2008) The Fourier-Kelvin Stellar Interferometer (FKSI): a review, progress report, and update. In: Optical and Infrared Interferometry, Proc. SPIE, vol 7013, p 70132Q, DOI $10.1117 / 12.790649$

Defrère D (2009) Detection of exozodiacal dust; a step toward earth-like planet characterization with infrared interferometry. $\mathrm{PhD}$ thesis, Liège University, Liège, Belgium

Defrère D, Absil O, Coudé du Foresto V, Danchi WC, den Hartog R (2008a) Nulling interferometry: performance comparison between space and ground-based sites for exozodiacal disc detection. A\&A490:435-445, DOI 10.1051/ 0004-6361:200810248, 0808.3713

Defrère D, Lay O, den Hartog R, Absil O (2008b) Earth-like planets: science performance predictions for future nulling interferometry missions. In: Optical and Infrared Interferometry, Proc. SPIE

Defrère D, Absil O, den Hartog R, Hanot C, Stark C (2010) Nulling interferometry: impact of exozodiacal clouds on the performance of future life-finding space missions. A\&A509:A9, DOI 10.1051/0004-6361/200912973, 0910. 3486

Defrère D, Hinz PM, Skemer AJ, Kennedy GM, Bailey VP, Hoffmann WF, Mennesson B, Millan-Gabet R, Danchi WC, Absil O, Arbo P, Beichman C, Brusa G, Bryden G, Downey EC, Durney O, Esposito S, Gaspar A, Grenz P, Haniff C, Hill JM, Lebreton J, Leisenring JM, Males JR, Marion L, McMahon TJ, Montoya M, Morzinski KM, Pinna E, Puglisi A, Rieke G, Roberge A, Serabyn E, Sosa R, Stapeldfeldt K, Su K, Vaitheeswaran V, Vaz A, Weinberger AJ, Wyatt MC (2015) First-light LBT Nulling Interferometric Observations: Warm Exozodiacal Dust Resolved within a Few AU of $\eta$ Crv. ApJ799:42, DOI 10.1088/0004-637X/799/1/42, 1501.04144 
Defrère D, Hinz PM, Mennesson B, Hoffmann WF, Millan-Gabet R, Skemer AJ, Bailey V, Danchi WC, Downey EC, Durney O, Grenz P, Hill JM, McMahon TJ, Montoya M, Spalding E, Vaz A, Absil O, Arbo P, Bailey H, Brusa G, Bryden G, Esposito S, Gaspar A, Haniff CA, Kennedy GM, Leisenring JM, Marion L, Nowak M, Pinna E, Powell K, Puglisi A, Rieke G, Roberge A, Serabyn E, Sosa R, Stapeldfeldt K, Su K, Weinberger AJ, Wyatt MC (2016) Nulling Data Reduction and On-sky Performance of the Large Binocular Telescope Interferometer. ApJ824:66, DOI 10.3847/0004-637X/824/2/66, 1601.06866

Des Marais DJ, Harwit MO, Jucks KW, Kasting JF, Lin DNC, Lunine JI, Schneider J, Seager S, Traub WA, Woolf NJ (2002) Remote Sensing of Planetary Properties and Biosignatures on Extrasolar Terrestrial Planets. Astrobiology 2:153-181, DOI 10.1089/15311070260192246

Eisenhauer F, Perrin G, Brandner W, Straubmeier C, Perraut K, Amorim A, Schöller M, Gillessen S, Kervella P, Benisty M, Araujo-Hauck C, Jocou L, Lima J, Jakob G, Haug M, Clénet Y, Henning T, Eckart A, Berger JP, Garcia P, Abuter R, Kellner S, Paumard T, Hippler S, Fischer S, Moulin T, Villate J, Avila G, Gräter A, Lacour S, Huber A, Wiest M, Nolot A, Carvas P, Dorn R, Pfuhl O, Gendron E, Kendrew S, Yazici S, Anton S, Jung Y, Thiel M, Choquet É, Klein R, Teixeira P, Gitton P, Moch D, Vincent F, Kudryavtseva N, Ströbele S, Sturm S, Fédou P, Lenzen R, Jolley P, Kister C, Lapeyrère V, Naranjo V, Lucuix C, Hofmann R, Chapron F, Neumann U, Mehrgan L, Hans O, Rousset G, Ramos J, Suarez M, Lederer R, Reess JM, Rohloff RR, Haguenauer P, Bartko H, Sevin A, Wagner K, Lizon JL, Rabien S, Collin C, Finger G, Davies R, Rouan D, Wittkowski M, Dodds-Eden K, Ziegler D, Cassaing F, Bonnet H, Casali M, Genzel R, Lena P (2011) GRAVITY: Observing the Universe in Motion. The Messenger $143: 16-24$

Enya K, Kataza H, Bierden P (2009) A Micro Electrical Mechanical Systems (MEMS)-based Cryogenic Deformable Mirror. PASP121:260-265, DOI 10.1086/598171

Errmann R, Minardi S, Labadie L, Muthusubramanian B, Dreisow F, Nolte S, Pertsch T (2015) Interferometric nulling of four channels with integrated optics. Appl Opt54:7449, DOI 10.1364/AO.54.007449

Fischer PD, Knutson HA, Sing DK, Henry GW, Williamson MW, Fortney JJ, Burrows AS, Kataria T, Nikolov N, Showman AP, Ballester GE, Désert JM, Aigrain S, Deming D, Lecavelier des Etangs A, Vidal-Madjar A (2016) HST Hot-Jupiter Transmission Spectral Survey: Clear Skies for Cool Saturn WASP-39b. ApJ827:19, DOI 10.3847/ 0004-637X/827/1/19, 1601.04761

Fridlund CVM (2000) Darwin - the Infrared Space Interferometer. In: Schürmann B (ed) Darwin and Astronomy : the Infrared Space Interferometer, ESA Special Publication, vol 451, p 11

Fridlund CVM (2004) The DARWIN project - An ESA cornerstone candidate mission. In: Penny A (ed) Planetary Systems in the Universe, IAU Symposium, vol 202, p 451

Fridlund CVM, d'Arcio L, den Hartog R, Karlsson A (2006) Status and recent progress of the Darwin mission in the Cosmic Vision program. In: Society of Photo-Optical Instrumentation Engineers (SPIE) Conference Series, Proceedings SPIE, vol 6268, p 62680R, DOI 10.1117/12.671040

Fridlund M, Gondoin P (2003) GENIE - The Darwin demonstrator. Ap\&SS286:93-98, DOI 10.1023/A: 1026166313620

Fridlund M, Eiroa C, Henning T, Herbst T, Lammer H, Léger A, Liseau R, Paresce F, Penny A, Quirrenbach A, Röttgering H, Selsis F, White GJ, Absil O, Defrère D, Schneider J, Tinetti G, Karlsson A, Gondoin P, den Hartog R, D'Arcio L, Stankov AM, Kilter M, Erd C, Beichman C, Coulter D, Danchi W, Devirian M, Johnston KJ, Lawson P, Lay OP, Lunine J, Kaltenegger L (2010) The Search for Worlds Like Our Own. Astrobiology 10:5-17, DOI 10.1089/ast.2009.0380

Gargaud M, Amils R, Quintanilla JC, Cleaves HJ, Irvine WM, Pinti DL, Viso M (2011) Encyclopedia of Astrobiology. DOI 10.1007/978-3-642-11274-4

Gómez-Leal I (2013) Spectrophotometry of the infrared emission of earth-like planets (phd thesis). PhD thesis, University of Bordeaux, France

Gondoin PA, Absil O, den Hartog RH, Wilhelm RC, Gitton PB, D' Arcio LL, Fabry P, Puech F, Fridlund MC, Schoeller M, Glindemann A, Bakker EJ, Karlsson AL, Peacock AJ, Volonte S, Paresce F, Richichi A (2004) Darwin-GENIE: a nulling instrument at the VLTI. In: Traub WA (ed) New Frontiers in Stellar Interferometry, Proc. SPIE, vol 5491, p 775, DOI 10.1117/12.549411

Grenfell JL, Grießmeier JM, Patzer B, Rauer H, Segura A, Stadelmann A, Stracke B, Titz R, Von Paris P (2007) Biomarker Response to Galactic Cosmic Ray-Induced $\mathrm{NO}_{x}$ And The Methane Greenhouse Effect in The Atmosphere of An Earth-Like Planet Orbiting An M Dwarf Star. Astrobiology 7:208-221, DOI 10.1089/ast.2006.0129, astro-ph/0702622 
Hanot C, Mennesson B, Martin S, Liewer K, Loya F, Mawet D, Riaud P, Absil O, Serabyn E (2011) Improving Interferometric Null Depth Measurements using Statistical Distributions: Theory and First Results with the Palomar Fiber Nuller. ApJ729:110, DOI 10.1088/0004-637X/729/2/110, 1103.4719

Hinz P, Bailey VP, Defrère D, Downey E, Esposito S, Hill J, Hoffmann WF, Leisenring J, Montoya M, McMahon T, Puglisi A, Skemer A, Skrutskie M, Vaitheeswaran V, Vaz A (2014) Commissioning the LBTI for use as a nulling interferometer and coherent imager. In: Optical and Infrared Interferometry IV, Proc. SPIE, vol 9146, p 91460T, DOI 10.1117/12.2057340

Hystad G, Downs RT, Grew ES, Hazen RM (2015) Statistical analysis of mineral diversity and distribution: Earth's mineralogy is unique. Earth and Planetary Science Letters 426:154-157, DOI 10.1016/j.epsl.2015.06.028

Jovanovic N, Martinache F, Guyon O, Clergeon C, Singh G, Kudo T, Garrel V, Newman K, Doughty D, Lozi J, Males J, Minowa Y, Hayano Y, Takato N, Morino J, Kuhn J, Serabyn E, Norris B, Tuthill P, Schworer G, Stewart P, Close L, Huby E, Perrin G, Lacour S, Gauchet L, Vievard S, Murakami N, Oshiyama F, Baba N, Matsuo T, Nishikawa J, Tamura M, Lai O, Marchis F, Duchene G, Kotani T, Woillez J (2015) The Subaru Coronagraphic Extreme Adaptive Optics System: Enabling High-Contrast Imaging on Solar-System Scales. PASP127:890, DOI 10.1086/682989, 1507.00017

Kaltenegger L, Traub WA, Jucks KW (2007) Spectral Evolution of an Earth-like Planet. ApJ658:598-616, DOI $10.1086 / 510996$, astro-ph/ 0609398

Kaltenegger L, Eiroa C, Fridlund CVM (2010) Target star catalogue for Darwin Nearby Stellar sample for a search for terrestrial planets. Ap\&SS326:233-247, DOI 10.1007/s10509-009-0223-3, 0810.5138

Karlsson AL, Wallner O, Perdigues Armengol JM, Absil O (2004) Three telescope nuller based on multibeam injection into single-mode waveguide. In: Traub WA (ed) Proc. SPIE, vol 5491, pp 831-+

Kitzmann D, Patzer ABC, von Paris P, Godolt M, Rauer H (2011) Clouds in the atmospheres of extrasolar planets. III. Impact of low and high-level clouds on the reflection spectra of Earth-like planets. A\&A534:A63, DOI 10.1051/ 0004-6361/201117375, 1108.3274

Kleidon A (2010) Life, hierarchy, and the thermodynamic machinery of planet Earth. Physics of Life Reviews 7:424460, DOI 10.1016/j.plrev.2010.10.002

Knutson HA, Charbonneau D, Allen LE, Fortney JJ, Agol E, Cowan NB, Showman AP, Cooper CS, Megeath ST (2007) A map of the day-night contrast of the extrasolar planet HD 189733b. Nature447:183-186, DOI 10.1038/ nature05782, 0705.0993

Krissansen-Totton J, Bergsman DS, Catling DC (2016) On Detecting Biospheres from Chemical Thermodynamic Disequilibrium in Planetary Atmospheres. Astrobiology 16:39-67, DOI 10.1089/ast.2015.1327, 1503.08249

Ksendzov A, Lay O, Martin S, Sanghera JS, Busse LE, Kim WH, Pureza PC, Nguyen VQ, Aggarwal ID (2007) Characterization of mid-infrared single mode fibers as modal filters. Appl Opt46:7957-7962, DOI 10.1364/AO.46. 007957

Ksendzov A, Lewi T, Lay OP, Martin SR, Gappinger RO, Lawson PR, Peters RD, Shalem S, Tsun A, Katzir A (2008) Modal filtering for midinfrared nulling interferometry using single mode silver halide fibers. Appl Opt47:5728, DOI 10.1364/AO.47.005728

Lawson P, Traub W (2006) Earth-Like Exoplanets: The Science of NASA's Navigator Program. JPL Publication

Lawson PR, Lay OP, Johnston KJ, Beichman CA (2007) Terrestrial Planet Finder Interferometer Science Working Group Report. NASA STI/Recon Technical Report N 8

Lay OP (2004) Systematic Errors in Nulling Interferometers. Appl Opt43:6100-6123, DOI 10.1364/AO.43.006100

Lay OP, Martin SR, Hunyadi SL (2007) Planet-finding performance of the TPF-I Emma architecture. In: Techniques and Instrumentation for Detection of Exoplanets III, Proc. SPIE, vol 6693, p 66930A, DOI 10.1117/12.732230

Le Bouquin JB, Berger JP, Lazareff B, Zins G, Haguenauer P, Jocou L, Kern P, Millan-Gabet R, Traub W, Absil O, Augereau JC, Benisty M, Blind N, Bonfils X, Bourget P, Delboulbe A, Feautrier P, Germain M, Gitton P, Gillier D, Kiekebusch M, Kluska J, Knudstrup J, Labeye P, Lizon JL, Monin JL, Magnard Y, Malbet F, Maurel D, Ménard F, Micallef M, Michaud L, Montagnier G, Morel S, Moulin T, Perraut K, Popovic D, Rabou P, Rochat S, Rojas C, Roussel F, Roux A, Stadler E, Stefl S, Tatulli E, Ventura N (2011) PIONIER: a 4-telescope visitor instrument at VLTI. A\&A535:A67, DOI 10.1051/0004-6361/201117586, 1109.1918

Lederberg J (1965) Signs of Life: Criterion-System of Exobiology. Nature207:9-13, DOI 10.1038/207009a0

Leger A, Pirre M, Marceau FJ (1993) Search for primitive life on a distant planet: relevance of 02 and 03 detections. A\&A277:309

Léger A, Mariotti JM, Mennesson B, Ollivier M, Puget JL, Rouan D, Schneider J (1996a) Could We Search for Primitive Life on Extrasolar Planets in the Near Future? Icarus123:249-255, DOI 10.1006/icar.1996.0155 
Léger A, Mariotti JM, Mennesson B, Ollivier M, Puget JL, Rouan D, Schneider J (1996b) The DARWIN project. Ap\&SS241:135-146, DOI 10.1007/BF00644221

Léger A, Selsis F, Sotin C, Guillot T, Despois D, Mawet D, Ollivier M, Labèque A, Valette C, Brachet F, Chazelas B, Lammer H (2004) A new family of planets? "Ocean-Planets". Icarus169:499-504, DOI 10.1016/j.icarus.2004.01. 001, astro-ph/0308324

Léger A, Fontecave M, Labeyrie A, Samuel B, Demangeon O, Valencia D (2011) Is the Presence of Oxygen on an Exoplanet a Reliable Biosignature? Astrobiology 11:335-341, DOI 10.1089/ast.2010.0516

Lovelock JE (1965) A Physical Basis for Life Detection Experiments. Nature207:568-570, DOI 10.1038/207568a0

Martin S, Booth A, Liewer K, Raouf N, Loya F, Tang H (2012) High performance testbed for four-beam infrared interferometric nulling and exoplanet detection. Appl Opt51:3907-3921, DOI 10.1364/AO.51.003907

Martin S, Serabyn G, Liewer K, Mennesson B (2017) Achromatic broadband nulling using a phase grating. Optica 4(1):110-113, DOI 10.1364/OPTICA.4.000110, URL http://www.osapublishing.org/optica/ abstract . cfm?URI $=$ opt ica-4-1-110

Martin SR, Booth AJ (2010) Demonstration of exoplanet detection using an infrared telescope array. A\&A520:A96, DOI 10.1051/0004-6361/201014942

Maurin AS, Selsis F, Hersant F, Belu A (2012) Thermal phase curves of nontransiting terrestrial exoplanets. II. Characterizing airless planets. A\&A538:A95, DOI 10.1051/0004-6361/201117054, 1110.3087

Mayor M, Queloz D (1995) A Jupiter-mass companion to a solar-type star. Nature378:355-359, DOI 10.1038/ $378355 \mathrm{a} 0$

Mennesson B, Mariotti JM (1997) Array Configurations for a Space Infrared Nulling Interferometer Dedicated to the Search for Earthlike Extrasolar Planets. Icarus128:202-212, DOI 10.1006/icar.1997.5731

Mennesson B, Ollivier M, Ruilier C (2002) Use of single-mode waveguides to correct the optical defects of a nulling interferometer. Journal of the Optical Society of America A 19:596-602, DOI 10.1364/JOSAA.19.000596

Mennesson B, Léger A, Ollivier M (2005) Direct detection and characterization of extrasolar planets: The Mariotti space interferometer. Icarus 178:570-588, DOI 10.1016/j.icarus.2005.05.012

Mennesson B, Hanot C, Serabyn E, Liewer K, Martin SR, Mawet D (2011a) High-contrast Stellar Observations within the Diffraction Limit at the Palomar Hale Telescope. ApJ743:178, DOI 10.1088/0004-637X/743/2/178

Mennesson B, Serabyn E, Hanot C, Martin SR, Liewer K, Mawet D (2011b) New Constraints on Companions and Dust within a Few AU of Vega. ApJ736:14, DOI 10.1088/0004-637X/736/1/14

Mennesson B, Millan-Gabet R, Serabyn E, Colavita MM, Absil O, Bryden G, Wyatt M, Danchi W, Defrère D, Doré O, Hinz P, Kuchner M, Ragland S, Scott N, Stapelfeldt K, Traub W, Woillez J (2014) Constraining the Exozodiacal Luminosity Function of Main-sequence Stars: Complete Results from the Keck Nuller Mid-infrared Surveys. ApJ797:119, DOI 10.1088/0004-637X/797/2/119

Mennesson B, Gaudi S, Seager S, Cahoy K, Domagal-Goldman S, Feinberg L, Guyon O, Kasdin J, Marois C, Mawet D, Tamura M, Mouillet D, Prusti T, Quirrenbach A, Robinson T, Rogers L, Scowen P, Somerville R, Stapelfeldt K, Stern D, Still M, Turnbull M, Booth J, Kiessling A, Kuan G, Warfield K (2016) The Habitable Exoplanet (HabEx) Imaging Mission: preliminary science drivers and technical requirements. In: Society of Photo-Optical Instrumentation Engineers (SPIE) Conference Series, Proceedings SPIE, vol 9904, p 99040L, DOI 10.1117/12.2240457

Monnier JD, Ireland MJ, Kraus S, Baron F, Creech-Eakman M, Dong R, Isella A, Merand A, Michael E, Minardi S, Mozurkewich D, Petrov R, Rinehart S, ten Brummelaar T, Vasisht G, Wishnow E, Young J, Zhu Z (2016) Architecture design study and technology road map for the Planet Formation Imager (PFI). In: Society of Photo-Optical Instrumentation Engineers (SPIE) Conference Series, Proceedings SPIE, vol 9907, p 99071O, DOI 10.1117/12.2233311, 1608.00580

Moskovitz NA, Gaidos E, Williams DM (2009) The Effect of Lunarlike Satellites on the Orbital Infrared Light Curves of Earth-Analog Planets. Astrobiology 9:269-277, DOI 10.1089/ast.2007.0209, 0810.2069

Ollivier M, Absil O, Allard F, Berger JP, Bordé P, Cassaing F, Chazelas B, Chelli A, Chesneau O, Coudé du Foresto V, Defrère D, Duchon P, Gabor P, Gay J, Herwats E, Jacquinod S, Kern P, Kervella P, Le Duigou JM, Léger A, Lopez B, Malbet F, Mourard D, Pelat D, Perrin G, Rabbia Y, Rouan D, Reiss JM, Rousset G, Selsis F, Stee P, Surdej J (2009) PEGASE, an infrared interferometer to study stellar environments and low mass companions around nearby stars. Experimental Astronomy 23:403-434, DOI 10.1007/s10686-008-9133-6

Owen T (1980) The Search for Early Forms of Life in Other Planetary Systems - Future Possibilities Afforded by Spectroscopic Techniques. In: Papagiannis MD (ed) Strategies for the Search for Life in the Universe, Astrophysics and Space Science Library, vol 83, p 177, DOI 10.1007/978-94-009-9115-6-17 
Peters RD, Lay OP, Lawson PR (2010) Mid-Infrared Adaptive Nulling for the Detection of Earthlike Exoplanets. PASP122:85-92, DOI 10.1086/649850

Quanz SP, Crossfield I, Meyer MR, Schmalzl E, Held J (2015) Direct detection of exoplanets in the 3-10 $\mu \mathrm{m}$ range with E-ELT/METIS. International Journal of Astrobiology 14:279-289, DOI 10.1017/S1473550414000135, 1404.0831

Rugheimer S, Kaltenegger L, Zsom A, Segura A, Sasselov D (2013) Spectral Fingerprints of Earth-like Planets Around FGK Stars. Astrobiology 13:251-269, DOI 10.1089/ast.2012.0888, 1212.2638

Sagan C, Thompson WR, Carlson R, Gurnett D, Hord C (1993) A search for life on Earth from the Galileo spacecraft. Nature365:715-721, DOI 10.1038/365715a0

Seager S, Bains W (2015) The search for signs of life on exoplanets at the interface of chemistry and planetary science. Science Advances 1:e1500,047-e1500,047, DOI 10.1126/sciadv.1500047

Seager S, Turner EL, Schafer J, Ford EB (2005) Vegetation's Red Edge: A Possible Spectroscopic Biosignature of Extraterrestrial Plants. Astrobiology 5:372-390, DOI 10.1089/ast.2005.5.372, astro-ph/ 0503302

Segura A, Krelove K, Kasting JF, Sommerlatt D, Meadows V, Crisp D, Cohen M, Mlawer E (2003) Ozone Concentrations and Ultraviolet Fluxes on Earth-Like Planets Around Other Stars. Astrobiology 3:689-708, DOI $10.1089 / 153110703322736024$

Segura A, Kasting JF, Meadows V, Cohen M, Scalo J, Crisp D, Butler RAH, Tinetti G (2005) Biosignatures from EarthLike Planets Around M Dwarfs. Astrobiology 5:706-725, DOI 10.1089/ast.2005.5.706, astro-ph / 0510224

Selsis F (2004) The Atmosphere of Terrestrial Exoplanets: Detection and Characterization. In: Beaulieu J, Lecavelier Des Etangs A, Terquem C (eds) Extrasolar Planets: Today and Tomorrow, Astronomical Society of the Pacific Conference Series, vol 321, p 170

Selsis F, Despois D, Parisot JP (2002) Signature of life on exoplanets: Can Darwin produce false positive detections? A\&A388:985-1003, DOI 10.1051/0004-6361:20020527

Selsis F, Wordsworth RD, Forget F (2011) Thermal phase curves of nontransiting terrestrial exoplanets. I. Characterizing atmospheres. A\&A532:A1, DOI 10.1051/0004-6361/201116654, 1104.4763

Selsis F, Maurin AS, Hersant F, Leconte J, Bolmont E, Raymond SN, Delbo' M (2013) The effect of rotation and tidal heating on the thermal lightcurves of super Mercuries. A\&A555:A51, DOI 10.1051/0004-6361/201321661, 1305.3858

Shao M, Unwin SC, Beichman C, Catanzarite J, Edberg SJ, Marr JC IV, Marcy G (2007) Finding Earth clones with SIM: The most promising near-term technique to detect, find masses for, and determine three-dimensional orbits of nearby habitable planets. In: Techniques and Instrumentation for Detection of Exoplanets III, Proceedings SPIE, vol 6693, p 66930C, DOI 10.1117/12.734671, 0704.0952

Simoncini E, Virgo N, Kleidon A (2013) Quantifying drivers of chemical disequilibrium: theory and application to methane in the Earth's atmosphere. Earth System Dynamics 4:317-331, DOI 10.5194/esd-4-317-2013

Snellen IAG, Brandl BR, de Kok RJ, Brogi M, Birkby J, Schwarz H (2014) Fast spin of the young extrasolar planet $\beta$ Pictoris b. Nature509:63-65, DOI 10.1038/nature13253

Traub WA, Kaltenegger L, Jucks KW, Turnbull MC (2006) Direct imaging of Earth-like planets from space (TPF-C). In: Society of Photo-Optical Instrumentation Engineers (SPIE) Conference Series, Proceedings SPIE, vol 6265, p 626502, DOI 10.1117/12.676257

Velusamy T, Angel RP, Eatchel A, Tenerelli D, Woolf NJ (2003) Single and double Bracewell nulling interferometer in space. In: Fridlund M, Henning T, Lacoste H (eds) Earths: DARWIN/TPF and the Search for Extrasolar Terrestrial Planets, ESA Special Publication, vol 539, pp 631-636

von Paris P, Cabrera J, Godolt M, Grenfell JL, Hedelt P, Rauer H, Schreier F, Stracke B (2011) Spectroscopic characterization of the atmospheres of potentially habitable planets: GL $581 \mathrm{~d}$ as a model case study. A\&A534:A26, DOI 10.1051/0004-6361/201117091, 1108.3670

von Paris P, Hedelt P, Selsis F, Schreier F, Trautmann T (2013) Characterization of potentially habitable planets: Retrieval of atmospheric and planetary properties from emission spectra. A\&A551:A120, DOI 10.1051/0004-6361/ 201220009, 1301.0217

Wallner O, Leeb WR, Winzer PJ (2002) Minimum length of a single-mode fiber spatial filter. Journal of the Optical Society of America A 19:2445-2448, DOI 10.1364/JOSAA.19.002445

Weber V, Barillot M, Haguenauer P, Kern PY, Schanen-Duport I, Labeye PR, Pujol L, Sodnik Z (2004) Nulling interferometer based on an integrated optics combiner. In: Traub WA (ed) New Frontiers in Stellar Interferometry, Proc. SPIE, vol 5491, p 842 
Woolf N, Angel JR (1998) Astronomical Searches for Earth-Like Planets and Signs of Life. ARA\&A36:507-538, DOI 10.1146/annurev.astro.36.1.507 